\title{
On Existence of Ground States in the Spin Boson Model
}

\author{
David Hasler, Benjamin Hinrichs $\mathbb{D}$, Oliver Siebert \\ Department of Mathematics, Friedrich Schiller University Jena, Ernst-Abbe-Platz 2, 07743 Jena, Germany. \\ E-mail: benjamin.hinrichs@uni-jena.de
}

Received: 25 March 2021 / Accepted: 23 July 2021

Published online: 9 August 2021 - (C) The Author(s) 2021

\begin{abstract}
We show the existence of ground states in the massless spin boson model without any infrared regularization. Our proof is non-perturbative and relies on a compactness argument. It works for arbitrary values of the coupling constant under the hypothesis that the second derivative of the ground state energy as a function of a constant external magnetic field is bounded.
\end{abstract}

\section{Introduction}

The spin boson model describes a quantum mechanical two-level system which is linearly coupled to a quantized field of bosons. If the bosons are relativistic and massless, the model is used as a simplified caricature describing an atom, coarsely approximated by two states, coupled to the quantized electromagnetic field. Although the model has been extensively investigated, see for example $[6,18,34]$ and references therein, it is still an active area of research, cf. $[8,14]$.

If this system has a ground state, i.e., if the infimum of the spectrum is an eigenvalue, this physically means that it exhibits binding. Furthermore, ground states are a necessary ingredient to study scattering theory in quantum field theories. In the case of massless bosons or photons in $\mathbb{R}^{d}$, we have the dispersion relation $\omega(k)=|k|$. As a consequence, the infimum of the spectrum is not isolated from the rest of the spectrum and establishing existence of a ground state is non-trivial. If one imposes a mild infrared regularization of the interaction function $f$, such that the quotient $f / \omega$ is square-integrable, e.g., in the case $d=3$ if we have $\delta>-1 / 2$ such that $f(k) \sim|k|^{\delta}$ for small photon momentum $|k|$, then existence of ground states has been shown $[5,6,10,11,18,30,33,34]$ and its analytic dependence on coupling parameters has been established [19]. However, in models of physical interest where $d=3$, the coupling function typically has the behavior $f(k) \sim|k|^{-1 / 2}$ and $f / \omega$ is no longer square-integrable. In such a situation the model is infrared-critical in the sense that an infrared problem may occur and a ground state ceases to exist. Such a behavior was most prominently observed for translation invariant 
models in [13,17,21], see also references therein. Moreover, the absence of ground states was shown for the Nelson model [30] as well as for generalized spin boson models [7], provided a nonvanishing expectation condition is satisfied. However, it may also happen in the infrared-critical case that the infrared divergences cancel and a ground state exists. Heuristically, the reason behind this cancellation is an underlying symmetry of the model. In particular, existence of ground states have been shown for models of non-relativistic quantum electrodynamics [9, 12,20,22,24,27]. Due to the absence of diagonal entries in the coupling matrix, Herbst and the first author [23] proved that the spin boson model does actually exhibit a ground state even in the infrared-critical case, see also [8] for a recent alternative proof providing new insight.

In this paper, we consider couplings which are more singular than in $[8,23]$ and prove the existence of a ground state in the spin boson model, e.g., in $d=3$ for any coupling $f(k) \sim|k|^{\delta}$ for $|k| \rightarrow 0$ with $\delta>-1$, provided an energy bound is satisfied. We note, this result is optimal in the sense that for $\delta=-1$ the field operator is no longer bounded in terms of the free field energy. In contrast to previous results, our result is non-perturbative and holds for all values of the coupling constant as long as the energy inequality holds. Let us be more precise on the statement. Denote by $\omega: \mathbb{R}^{d} \rightarrow[0, \infty)$ the boson dispersion relation and $f: \mathbb{R}^{d} \rightarrow \mathbb{R}$ the interaction of the quantum field and the two-level system. Then, the lower-bounded and self-adjoint Hamilton operator describing the spin boson model acts on the Hilbert space $\mathbb{C}^{2} \otimes \mathcal{F}$, with $\mathcal{F}$ being the usual Fock space on $L^{2}\left(\mathbb{R}^{d}\right)$, and is given as

$$
H(\omega, f)=\sigma_{z} \otimes \mathbb{1}+\mathbb{1} \otimes \mathrm{d} \Gamma(\omega)+\int_{\mathbb{R}^{d}} f(k) \sigma_{x} \otimes\left(a_{k}^{\dagger}+a_{k}\right) \mathrm{d} k .
$$

Here, $\mathrm{d} \Gamma(\omega)$ denotes the second quantization of the operator of multiplication by $\omega$, moreover $a_{k}, a_{k}^{\dagger}$ are the distributions describing annihilation and creation operators, respectively, and $\sigma_{x}$ and $\sigma_{z}$ denote the Pauli matrices. A more rigorous definition can be found in Sect. 2. For the energy inequality we consider $e_{n}(\mu)=\inf \sigma\left(H\left(\omega_{n}, f\right)+\right.$ $\left.\mu\left(\sigma_{x} \otimes \mathbb{1}\right)\right)$, where the sequence $\left(\omega_{n}\right)_{n \in \mathbb{N}}$ converges to $\omega$ uniformly and is chosen, such that $f / \omega_{n}$ is square-integrable. The parameter $\mu \in \mathbb{R}$ can hereby by interpreted as an external magnetic field. Explicitly, we assume that the second derivative of $e_{n}(\mu)$ exists at $\mu=0$ and is bounded as $n \rightarrow \infty$, for our result to hold. Using a Feynman-Kac-Nelson type formula [26], this assumption is related to a bound on the magnetic susceptibility of a continuous Ising model. We note that such a bound has been shown in our situation for the case $d=3$ and $\delta=-1 / 2$ [33]. For $d=3$ and $\delta \in(-1,-1 / 2)$, the bound has been shown to hold for the discrete Ising model, cf. [15] and references therein. We give a proof of our assumption by taking a continuum limit of the discrete Ising model in [25]. As a consequence of our result, non-existence of a ground state for large coupling would imply the divergence of the magnetic susceptibility in the corresponding Ising model.

For the proof of our result, we utilize that the existence of ground states for the infrared regular situation has been established using a variety of techniques. Hence, if we consider $H\left(\omega_{n}, f\right)$ as above, then a ground state $\psi_{n}$ exists. We then prove these ground states lie in a compact set and hence there exists a strongly convergent subsequence $\left(\psi_{n_{k}}\right)_{k \in \mathbb{N}}$. The limit of this sequence will be the ground state of $H(\omega, f)$. 


\section{Model and Statement of Results}

Throughout this paper we assume $d \in \mathbb{N}$ and write $\mathfrak{h}=L^{2}\left(\mathbb{R}^{d}\right)$ for the state space of a single boson. Then, let $\mathcal{F}$ be the bosonic Fock space defined by

$$
\mathcal{F}=\mathbb{C} \oplus \bigoplus_{n=1}^{\infty} \mathcal{F}^{(n)} \quad \text { with } \mathcal{F}^{(n)}=L_{\text {sym }}^{2}\left(\mathbb{R}^{n d}\right),
$$

where we symmetrize over the $n \mathbb{R}^{d}$-variables in each component. We write an element $\psi \in \mathcal{F}$ as $\psi=\left(\psi^{(n)}\right)_{n \in \mathbb{N}_{0}}$ and define the vacuum $\Omega=(1,0,0, \ldots)$.

For a measurable function $\omega: \mathbb{R}^{d} \rightarrow \mathbb{R}$, we define

$$
\mathrm{d} \Gamma(\omega)=0 \oplus \bigoplus_{n=1}^{\infty} \omega^{(n)} \quad \text { with } \omega^{(n)}\left(k_{1}, \ldots, k_{n}\right)=\sum_{i=1}^{n} \omega\left(k_{i}\right)
$$

as operators on $\mathcal{F}$. Further, for $f \in \mathfrak{h}$, we define the annihilation operator $a(f)$ and creation operator $a^{\dagger}(f)$ using $a(f) \Omega=0, a^{\dagger}(f) \Omega=f$ and for $g \in \mathcal{F}^{(n)}$

$$
\begin{aligned}
(a(f) g)\left(k_{1}, \ldots, k_{n-1}\right) & =\sqrt{n} \int \overline{f(k)} g\left(k, k_{1}, \ldots, k_{n-1}\right) \mathrm{d} k \in \mathcal{F}^{(n-1)} \\
\left(a^{\dagger}(f) g\right)\left(k_{1}, \ldots, k_{n}, k_{n+1}\right) & =\frac{1}{\sqrt{n+1}} \sum_{i=1}^{n+1} f\left(k_{i}\right) g\left(k_{1}, \ldots, \widehat{k}_{i}, \ldots, k_{n+1}\right) \in \mathcal{F}^{(n+1)},
\end{aligned}
$$

where $\widehat{k}_{i}$ means that $k_{i}$ is omitted from the argument. One can show that these operators can be extended to closed operators on $\mathcal{F}$ that satisfy $(a(f))^{*}=a^{\dagger}(f)$. From the creation and annihilation operator, we define the field operator

$$
\varphi(f)=\overline{a(f)+a^{\dagger}(f)} .
$$

The following properties are well-known and can for example be found in [4,31].

Lemma 2.1. Let $\omega, \omega^{\prime}: \mathbb{R}^{d} \rightarrow \mathbb{R}$ and $f \in \mathfrak{h}$. Then

(i) $\mathrm{d} \Gamma(\omega)$ and $\varphi(f)$ are self-adjoint.

(ii) If $\omega^{\prime} \geq \omega$, then $\mathrm{d} \Gamma\left(\omega^{\prime}\right) \geq \mathrm{d} \Gamma(\omega)$. Especially, if $\omega \geq 0$, then $\mathrm{d} \Gamma(\omega) \geq 0$.

(iii) Assume $\omega>0$ almost everywhere and $\omega^{-\frac{1}{2}} f \in \mathfrak{h}$. Then $\varphi(f)$ and $a(f)$ are $\mathrm{d} \Gamma(\omega)^{1 / 2}$-bounded and for $\psi \in \mathcal{D}\left(\mathrm{d} \Gamma(\omega)^{1 / 2}\right)$ we have

$$
\begin{aligned}
& \|a(f) \psi\| \leq\left\|\omega^{-\frac{1}{2}} f\right\|\left\|\mathrm{d} \Gamma(\omega)^{\frac{1}{2}} \psi\right\| \text { and } \\
& \|\varphi(f) \psi\| \leq 2\left\|\left(\omega^{-\frac{1}{2}}+1\right) g\right\|\left\|(\mathrm{d} \Gamma(\omega)+1)^{\frac{1}{2}} \psi\right\| .
\end{aligned}
$$

In particular, $\varphi(g)$ is infinitesimally $\mathrm{d} \Gamma(\omega)$-bounded.

Now, let

$$
\mathcal{H}=\mathbb{C}^{2} \otimes \mathcal{F} \cong \mathcal{F} \oplus \mathcal{F}
$$


where the unitary equivalence is implemented by $\left(v_{1}, v_{2}\right) \otimes \psi \mapsto v_{1} \psi \oplus v_{2} \psi$. Furthermore, let $\sigma_{x}$ and $\sigma_{z}$ be the usual $2 \times 2$ Pauli-matrices

$$
\sigma_{x}=\left(\begin{array}{ll}
0 & 1 \\
1 & 0
\end{array}\right) \quad \text { and } \quad \sigma_{z}=\left(\begin{array}{cc}
1 & 0 \\
0 & -1
\end{array}\right) .
$$

For a measurable function $\omega: \mathbb{R}^{d} \rightarrow \mathbb{R}$ and $f \in \mathfrak{h}$, we define the spin boson Hamiltonian on $\mathcal{H}$ as

$$
H(\omega, f)=\sigma_{z} \otimes \mathbb{1}+\mathbb{1} \otimes \mathrm{d} \Gamma(\omega)+\sigma_{x} \otimes \varphi(f) .
$$

Lemma 2.2. Assume that $\omega: \mathbb{R}^{d} \rightarrow \mathbb{R}$ is measurable and almost everywhere positive, $f \in \mathfrak{h}, \omega^{-1 / 2} f \in \mathfrak{h}$, and $\mu \in \mathbb{R}$. Then $H(\omega, f)+\mu \sigma_{x} \otimes \mathbb{1}$ defines a lower-bounded self-adjoint operator on the domain $\mathcal{D}(\mathbb{1} \otimes \mathrm{d} \Gamma(\omega))$. Any core for $\mathbb{1} \otimes \mathrm{d} \Gamma(\omega)$ is a core for $H(\omega, f)+\mu \sigma_{x} \otimes \mathbb{1}$.

Proof. The operator $K=\sigma_{z} \otimes \mathbb{1}+\mathbb{1} \otimes \mathrm{d} \Gamma(\omega)+\mu \sigma_{x} \otimes \mathbb{1}$ is self-adjoint as a sum of a self-adjoint operator with bounded self-adjoint operators and has domain $\mathcal{D}(K)=$ $\mathcal{D}(1 \otimes \mathrm{d} \Gamma(\omega))$. Moreover, it is bounded from below since $\mathrm{d} \Gamma(\omega)$ is non-negative by Lemma 2.1 and $\sigma_{x}, \sigma_{z}$ are bounded. The symmetric operator $\sigma_{x} \otimes \varphi(f)$ is infinitesimally $K$-bounded by Lemma 2.1. Hence, the statement follows from the Kato-Rellich theorem (cf. [31, Theorem X.12]).

From now on, we fix a measurable non-negative function $\omega: \mathbb{R}^{d} \rightarrow \mathbb{R}$ and an $f \in \mathfrak{h}$ and work under the following assumptions.

\section{Hypothesis A.}

(i) $\omega$ takes positive values almost everywhere,

(ii) $\omega(k) \stackrel{|k| \rightarrow \infty}{\longrightarrow} \infty$.

(iii) There exists $\alpha_{1}>0$, such that $\omega$ is locally $\alpha_{1}$-Hölder continuous.

(iv) There exists $\epsilon>0$, such that $\omega^{-1 / 2} f \in L^{2}\left(\mathbb{R}^{d}\right) \cap L^{2+\epsilon}\left(\mathbb{R}^{d}\right)$.

(v) There exists $\alpha_{2}>0$, such that

$$
\sup _{|p| \leq 1} \int_{\mathbb{R}^{d}} \frac{|f(k+p)-f(k)|}{\sqrt{\omega(k)}|p|^{\alpha_{2}}} \mathrm{~d} k<\infty .
$$

(vi) We have

$$
\sup _{|p| \leq 1} \int_{\mathbb{R}^{d}} \frac{|f(k)|}{\sqrt{\omega(k)} \omega(k+p)} \mathrm{d} k<\infty .
$$

Example 2.3. In $d=3$ dimensions elementary estimates show that the assumptions of Hypothesis A hold for the choices

$$
\omega(k)=|k|, \quad f(k)=g \kappa(k)|k|^{\delta}
$$

for any $\delta>-1, g \in \mathbb{R}$, and a cutoff function $\kappa$ of the form $\kappa(k)=1_{|k| \leq \Lambda}$, for some $\Lambda>0$, or $\kappa(k)=\exp \left(-c k^{2}\right)$, for some $c>0$. The number $g$ will be referred to as the coupling parameter. 
Example 2.4. More generally as in Example 2.3, we consider, for $k \in \mathbb{R}^{d}$, the functions $\omega(k)=|k|^{\alpha}, f(k)=g \kappa(k)|k|^{\beta}$, with some $\alpha>0, \beta \in \mathbb{R}, g \in \mathbb{R}$, and $\kappa$ a cutoff function as in Example 2.3. Then Hypothesis A holds under the condition $d>\max \{\alpha-$ $\left.2 \beta, \frac{3}{2} \alpha-\beta, \frac{1}{2} \alpha-2 \beta\right\}$.

The second assumption we need is a differentiability condition for the infimum of the spectrum with respect to the constant magnetic field $\mu$. For this, we need to ensure that $\omega$ can be approximated by a family of functions which are bounded from below by some positive constant.

Hypothesis B. There exists a decreasing sequence $\left(\omega_{n}\right)_{n \in \mathbb{N}}$ of nonnegative measurable functions $\omega_{n}: \mathbb{R}^{d} \rightarrow \mathbb{R}$ converging uniformly to $\omega$, with the following properties.

(i) There exists $\alpha_{1}>0$, such that $\omega_{n}$ is locally $\alpha_{1}$-Hölder continuous for all $n \in \mathbb{N}$.

(ii) $\inf _{k \in \mathbb{R}^{d}} \omega_{n}(k)>0$.

(iii) The function $e_{n}(\mu)=\inf \sigma\left(H\left(\omega_{n}, f\right)+\mu \sigma_{x} \otimes \mathbb{1}\right)$ is twice differentiable at zero and

$$
C_{\chi}:=\sup _{n \in \mathbb{N}}\left(-e_{n}^{\prime \prime}(0)\right)<\infty .
$$

Remark 2.5. We note that (i) and (ii) of Hypothesis B are satisfied for the typical choice of a massive photon dispersion relation

$$
\omega_{n}=\sqrt{m_{n}^{2}+\omega^{2}}
$$

or also $\omega_{n}=\omega+m_{n}$, where $\left(m_{n}\right)_{n \in \mathbb{N}}$ is any sequence of positive numbers decreasing monotonically to zero. The constant $m_{n}$ can be understood to be a photon mass. The result we prove is, however, independent of the specific choice of $\omega_{n}$.

Remark 2.6. The differentiability assumption in Hypothesis B (iii) can be shown to hold by regular analytic perturbation theory provided (ii) holds, since (ii) implies that the ground state energy is separated from the rest of the spectrum (cf. [5,6] or Proposition 3.2). However, the uniform bound on the second derivative is nontrivial to establish. We note that this assumption will be translated into a bound on the resolvent by means of second order perturbation theory, see Lemma 4.3. In fact, this bound on the resolvent is what we need in the proof of the main result, i.e., Theorem 2.8 holds if one replaces (iii) by the bound in Lemma 4.3.

Remark 2.7. Let us discuss (2.10) in $d=3$ dimensions for the case given in (2.9) and (2.11). If $\delta>-1 / 2$ then (2.10) follows, e.g., from the estimates in [19]. In the case $\delta=-1 / 2$, (2.9) has been shown for small values of the coupling constant in [33], using that ground state properties of the spin boson Hamiltonian are related to the correlation functions of a continuous one dimensional Ising model with long range interaction, cf. [35] and [3]. In particular, (2.10) translates to the corresponding Ising model having finite magnetic suszeptibility. For $\delta \in(-1 / 2,-1)$ the finiteness of the magnetic suszeptibility has been shown for the discrete Ising model, [15]. In fact, for $\delta \in[-1 / 2,-1)$ the bound (2.10) does not hold anymore for large values of the coupling constant. This follows from the relation to the Ising model and the phase transition for Ising models with coupling decaying quadratically in the distance, $\mathrm{cf}$. [3,28,33]. On the other hand, for small couplings, using the relation to a continuous Ising model, it has been shown in [1] that $g \mapsto \inf \sigma(H(|\cdot|, g f))$ is analytic in a neighborhood of zero if $f, f|\cdot|^{-1 / 2} \in \mathfrak{h}$, which corresponds to $\delta>-1$. Note that the infimum of the spectrum can be analytic although there does not exist a ground state (cf. [2]). Thus, it is not unreasonable to suspect that (2.10) might in fact hold for $\delta>-1$ provided the coupling is sufficiently small. 
Our main result now is the following.

Theorem 2.8. Assume Hypothesis A and $\mathrm{B}$ hold. Then inf $\sigma(H(\omega, f))$ is an eigenvalue of $H(\omega, f)$.

Remark 2.9. This result has been proven for infrared regular models, e.g., under the additional assumption inf $\operatorname{k\in \mathbb {R}}^{d} \omega(k)>0$ in [5] (see [6] for a generalization of this result) and under the assumption $\omega^{-1} f \in \mathfrak{h}$ in [18], which in $d=3$ corresponds to $\delta>-1 / 2$ in (2.9). Specifically for $d=3$ the existence has been shown in situations where $\omega^{-1} f \notin \mathfrak{h}$ in $[8,23]$. The results in these papers include the case (2.9) with $\delta=-1 / 2$ provided the coupling $g$ is sufficiently small. The results are perturbative in nature and were obtained using operator theoretic renormalization and iterated perturbation theory, respectively. In particular, [23] not only shows existence, but also analyticity of the ground state and the ground state energy in the coupling constant. Concerning existence, the result of Theorem 2.8 goes beyond. It shows existence for any $\delta>-1$ and arbitrary coupling, as long as the derivative bound (2.10) is finite.

Remark 2.10. In [33] finite temperature KMS states of the spin boson Hamiltonian where investigated for $\int_{\mathbb{R}^{d}} f(k)^{2} e^{-\omega(k)|t|} \mathrm{d} k \cong t^{-2}$ for large $t$. For $d=3$ this corresponds to $\delta=-1 / 2$ in (2.9). Using results about the one dimensional continuous Ising model, it was established that the KMS states have a weak limit as the temperature drops to zero. In particular, it was shown that there exists a critical coupling such that the expectation of the number of bosons is finite below and infinite at and above the critical coupling strength. We note that for the proof of the main theorem we use a similar bound on the number of photons, see Lemma 4.5 (i) (which is in fact weaker than the one in [33]).

Remark 2.11. We note that our result gives a physically explicit bound on the coupling constant via (2.10), where the left hand side of (2.10) is proportional to the magnetic suszeptibility of the corresponding Ising model. As a consequence of Theorem 2.8 the absence of a ground state implies that the magnetic suszeptibility must diverge. Given the existence results in $[8,23]$, in case $\delta=-1 / 2$ in (2.9), the absence of a ground state for large coupling could provide an alternative method of proof for phase transitions in continuous long range Ising models. To the best of our knowledge the absence of a ground state in the spin boson model with $\mu=0$ for $\delta \in(-1,-1 / 2]$ and large coupling has not yet been shown. Nevertheless, we refer the reader to results $[14,33]$ where the large coupling limit has been investigated.

The method of proof we use is based on the proof in [20]. It was applied to the infraredcritical model of non-relativistic quantum electrodynamics by two of the authors in [27].

For the proof of Theorem 2.8, we denote by $\psi_{n}$ the ground state of $H\left(\omega_{n}, f\right)$, which exists due to the assumption $\inf _{k \in \mathbb{R}^{d}} \omega_{n}>0$. We then prove, that all of them lie in a compact set $K \subset \mathbb{C}^{2} \otimes \mathcal{F}$. Hence, there exists a subsequence $\left(\psi_{n_{j}}\right)_{j \in \mathbb{N}}$ converging strongly to some $\psi \in K$. It then remains to show that $\psi \neq 0$ actually is a ground state of $H(\omega, f)$.

The rest of this paper is organized as follows. In Sect. 3, we show some simple properties of the states $\psi_{n}$ and the corresponding ground state energies. In Sect. 4, we then derive necessary upper bounds with respect to the photon number to construct the compact set $K$ in Sect. 5.

\section{Ground State Properties for Massive Photons}

In this section, we derive some simple properties of the ground state energy of the infrared regular spin boson Hamiltonian. Throughout this section we will assume Hypothesis A 
and that the sequence $\left(\omega_{n}\right)_{n \in \mathbb{N}}$ is chosen as in Hypothesis B (ii). We set

$$
H=H(\omega, f) \text { and } H_{n}=H\left(\omega_{n}, f\right),
$$

as well as $E=\inf \sigma(H)$ and $E_{n}=\inf \sigma\left(H_{n}\right)$ for all $n \in \mathbb{N}$.

\section{Lemma 3.1. We have}

(i) $H \leq H_{n^{\prime}} \leq H_{n}$ for $n \leq n^{\prime}$,

(ii) $\lim _{n \rightarrow \infty} E_{n}=E$.

Proof. (i) follows from the monotonicity of $\left(\omega_{n}\right)$ and Lemma 2.1. We set $N=\mathbb{1} \otimes \mathrm{d} \Gamma(1)$. Then, due to the uniform convergence of $\left(\omega_{n}\right)$, there is a sequence $\left(C_{n}\right) \subset \mathbb{R}^{+}$satisfying $C_{n} \stackrel{n \rightarrow \infty}{\longrightarrow} 0$ and $\omega_{n} \leq \omega+C_{n}$. Hence,

$$
\mathrm{d} \Gamma\left(\omega_{n}\right) \leq \mathrm{d} \Gamma(\omega)+C_{n} \mathrm{~d} \Gamma(1), \quad \text { which implies } \quad H_{n} \leq H+C_{n} N
$$

On the other hand let $\varepsilon>0$ and fix $\varphi_{\varepsilon} \in \mathcal{D}(N) \cap \mathcal{D}\left(H_{0}\right)$ with $\left\|\varphi_{\varepsilon}\right\|=1$, such that

$$
\left\langle\varphi_{\varepsilon}, H \varphi_{\varepsilon}\right\rangle \leq E+\varepsilon
$$

This is possible, since $\mathcal{D}(N) \cap \mathcal{D}\left(H_{0}\right)$ is a core for $\mathbb{1} \otimes \mathrm{d} \Gamma(\omega)$ and hence for $H$, by Lemma 2.2. Together with (i), we obtain

$$
\begin{aligned}
E \leq E_{n} & \leq\left\langle\varphi_{\varepsilon}, H_{n} \varphi_{\varepsilon}\right\rangle \leq\left\langle\varphi_{\varepsilon}, H \varphi_{\varepsilon}\right\rangle+C_{n}\left\langle\varphi_{\varepsilon}, N \varphi_{\varepsilon}\right\rangle \\
& \leq E+\varepsilon+C_{n}\left\langle\varphi_{\varepsilon}, N \varphi_{\varepsilon}\right\rangle \stackrel{n \rightarrow \infty}{\longrightarrow} E+\varepsilon .
\end{aligned}
$$

Now (ii) follows in the limit $\varepsilon \rightarrow 0$.

As mentioned above the bound Hypothesis B (ii) implies the existence of a ground state, which is the content of the following proposition.

Proposition 3.2. For all $n \in \mathbb{N}, E_{n}$ is a simple eigenvalue of $H_{n}$.

Further, $\left[E_{n}, E_{n}+\inf _{k \in \mathbb{R}^{d}} \omega_{n}(k)\right) \cap \sigma_{\mathrm{ess}}\left(H_{n}\right)=\emptyset$.

Proof. The existence has been shown in [5] and the uniqueness for arbitrary couplings has been shown in [23], see also [17].

Let $\psi_{n}$ be a normalized eigenvector of $H_{n}$ to the eigenvalue $E_{n}$. A main ingredient of our proof then is the following proposition.

Proposition 3.3. The sequence $\left(\psi_{n}\right)_{n \in \mathbb{N}}$ is minimizing for $H$, i.e.,

$$
0 \leq\left\langle\psi_{n},(H-E) \psi_{n}\right\rangle \stackrel{n \rightarrow \infty}{\longrightarrow} 0 .
$$

Proof. We use Lemma 3.1 and find

$$
0 \leq\left\langle\psi_{n},(H-E) \psi_{n}\right\rangle \leq\left\langle\psi_{n},\left(H_{n}-E\right) \psi_{n}\right\rangle=E_{n}-E \rightarrow 0 .
$$




\section{Infrared Bounds}

In this section we derive essential bounds on the ground states $\psi_{n}$, which are uniform in $n \in \mathbb{N}$. Throughout this section we will assume that Hypothesis A and B hold. We recall the following definition in Hypothesis B

$$
e_{n}(\mu)=\inf \sigma\left(H\left(\omega_{n}, f\right)+\mu \sigma_{x} \otimes \mathbb{1}\right) \text { for } n \in \mathbb{N} \text { and } \mu \in \mathbb{R} .
$$

Note that by the definitions in (3.1), we have $E_{n}=e_{n}(0)$. The next Lemma is a simple symmetry argument.

Lemma 4.1. We have $e_{n}(\mu)=e_{n}(-\mu)$ for all $n \in \mathbb{N}$.

Proof. We define the unitary operator $U=e^{\mathrm{i} \frac{\pi}{2} \sigma_{z}} \otimes(-1)^{\mathrm{d} \Gamma(1)}$. It easily follows from the definitions that $e^{\mathrm{i} \frac{\pi}{2} \sigma_{z}} \sigma_{x} e^{-\mathrm{i} \frac{\pi}{2} \sigma_{z}}=-\sigma_{x}$ and $(-1)^{\mathrm{d} \Gamma(1)} \varphi(f)(-1)^{\mathrm{d} \Gamma(1)}=\varphi(-f)$. Now, using that $\mathrm{d} \Gamma$-operators commute, we obtain $U\left(H\left(\omega_{n}, f\right)+\mu \sigma_{x} \otimes \mathbb{1}\right) U^{*}=H\left(\omega_{n}, f\right)-$ $\mu \sigma_{x} \otimes \mathbb{1}$, which proves the claim.

Now, let $\psi^{(\ell+1)} \in \mathcal{F}^{(\ell+1)}$ for some $\ell \in \mathbb{N}$. Then the map $k \mapsto \psi^{(\ell+1)}(k, \cdots)$ is an element of $L^{2}\left(\mathbb{R}^{d}, \mathcal{F}^{(\ell)}\right)$, by the Fubini-Tonelli theorem (cf. [16, Theorem 2.37]). Hence, the prescription

$$
\left(a_{k} \psi^{(\ell+1)}\right)\left(k_{1}, \ldots, k_{\ell}\right)=\sqrt{\ell+1} \psi^{(\ell+1)}\left(k, k_{1}, \ldots, k_{\ell}\right) .
$$

yields a well-defined element $a_{k} \psi^{(\ell+1)} \in \mathcal{F}^{(\ell)}$ for almost every $k \in \mathbb{R}^{d}$. Further, for $n \in \mathbb{N}$, we define the operator

$$
R_{n}(k)=\left(H_{n}-E_{n}+\omega_{n}(k)\right)^{-1} \text { for } k \in \mathbb{R}^{d},
$$

which is bounded by Hypothesis B, and the spectral theorem directly yields

$$
\left\|R_{n}(k)\right\| \leq \frac{1}{\omega_{n}(k)} .
$$

The next statement is well-known and can be found under the name pull-through formula throughout the literature, cf. [10,18]. In the statement we write $\psi_{n}=\left(\psi_{n, 1}, \psi_{n, 2}\right)$ in the sense of (2.6) and denote

$$
a_{k} \psi_{n}=\left(a_{k} \psi_{n, 1}, a_{k} \psi_{n, 2}\right) \text { and } \sigma_{x} \psi_{n}=\left(\sigma_{x} \otimes \mathbb{1}\right) \psi_{n}=\left(\psi_{n, 2}, \psi_{n, 1}\right) .
$$

Lemma 4.2. Let $n \in \mathbb{N}$. Then, for almost every $k \in \mathbb{R}^{d}$, the vector $a_{k} \psi_{n} \in \mathcal{H}$ and

$$
a_{k} \psi_{n}=-f(k) R_{n}(k) \sigma_{x} \psi_{n} .
$$

The infrared bounds we want to obtain in this section are bounds on $R_{n}(k) \sigma_{x} \psi_{n}$. To that end, we start by translating Hypothesis B into a resolvent bound.

Lemma 4.3. For all $n \in \mathbb{N}$, we have $\left\langle\psi_{n}, \sigma_{x} \psi_{n}\right\rangle=0$ and

$$
0 \leq\left\langle\sigma_{x} \psi_{n},\left(H_{n}-E_{n}\right)^{-1} \sigma_{x} \psi_{n}\right\rangle=-\frac{1}{2} e_{n}^{\prime \prime}(0) .
$$


Proof. The proof uses second order analytic perturbation theory, for details see $[29,32]$. The operator valued function $\eta \mapsto H_{n}(\eta):=H_{n}+\eta \sigma_{x} \otimes \mathbb{1}$ defines an analytic family of type (A) for $\eta \in \mathbb{C}$ (cf. [29, Chapter VII, Theorem 2.6]). By Proposition 3.2, we know that $e_{n}(0)$ is a non-degenerate eigenvalue of $H_{n}(0)$ in the discrete spectrum. Hence, in some ball around $\eta=0$ there exists a unique analytic function $e_{n}(\cdot)$ and a $\mathcal{D}\left(H_{n}\right)$-valued analytic function $\phi_{n}(\cdot)$, such that $\phi_{n}(0)=\psi_{n}$ and

$$
H_{n}(\eta) \phi_{n}(\eta)=e_{n}(\eta) \phi_{n}(\eta)
$$

cf. [32, Theorem XII.8]. Thus, $e_{n}(0)=E_{n}$.

The first derivative of (4.6) yields $H_{n}^{\prime}(0) \psi_{n}+H_{n} \phi_{n}^{\prime}(0)=e_{n}^{\prime}(0) \psi_{n}+E_{n} \phi_{n}^{\prime}(0)$. Multiplying $\psi_{n}$ from the left and using $\left\|\psi_{n}\right\|=1$ as well as $H_{n}^{\prime}(0)=\sigma_{x} \otimes \mathbb{1}$ yields $e_{n}^{\prime}(0)=\left\langle\psi_{n}, \sigma_{x} \psi_{n}\right\rangle$. Hence, Lemma 4.1 implies $\left\langle\psi_{n}, \sigma_{x} \psi_{n}\right\rangle=0$. Using that $e_{n}^{\prime}(0)=0$, we can solve for the first derivative of the eigenvector and obtain $\phi_{n}^{\prime}(0)=$ $-\left(H_{n}-E_{n}\right)^{-1} \sigma_{x} \psi_{n}+\alpha \psi_{n}$ for some $\alpha \in \mathbb{C}$. Now, taking the second derivative of (4.6) and using $H_{n}^{\prime \prime}(0)=0$, we similarly obtain $e_{n}^{\prime \prime}(0)=2\left\langle\psi_{n}, \sigma_{x} \phi_{n}^{\prime}(0)\right\rangle$. Inserting the first derivative of the eigenvector, we obtain the statement.

This gives us the required infrared bound.

Lemma 4.4. We have $\left\|R_{n}(k) \sigma_{x} \psi_{m}\right\| \leq \sqrt{\frac{-e_{n}^{\prime \prime}(0)}{\omega_{n}(k)}}$ for all $n \in \mathbb{N}$.

Proof. By the product inequality, we have

$$
\left\|R_{n}(k) \sigma_{x} \psi_{n}\right\| \leq\left\|R_{n}(k)\left(H_{n}-E_{n}\right)^{1 / 2}\right\|\left\|\left(H_{n}-E_{n}\right)^{-1 / 2} \sigma_{x} \psi_{n}\right\| .
$$

By Lemma 4.3, the second factor on the right hand side can be estimated using

$$
\left\|\left(H_{n}-E_{n}\right)^{-1 / 2} \sigma_{x} \psi_{n}\right\| \leq \sqrt{-e_{n}^{\prime \prime}(0)} .
$$

It remains to estimate the first factor in (4.7). Using $\left\|R_{n}(k)^{1 / 2}\left(H_{n}-E_{n}\right)^{1 / 2}\right\| \leq 1$ we find with (4.4)

$$
\left\|R_{n}(k)\left(H_{n}-E_{n}\right)^{1 / 2}\right\| \leq\left\|R_{n}(k)^{1 / 2}\right\| \leq \frac{1}{\sqrt{\omega_{n}(k)}}
$$

We combine this result with the pull-through formula. To that end let $C_{\chi}$ be defined as in Hypothesis B (iii).

Lemma 4.5. Let $B_{1}=\left\{x \in \mathbb{R}^{d}:|x| \leq 1\right\}$.

(i) For all $n \in \mathbb{N}$ and almost all $k \in \mathbb{R}^{d}$, we have $\left\|a_{k} \psi_{n}\right\| \leq \frac{|f(k)|}{\sqrt{\omega(k)}} C_{\chi}^{1 / 2}$.

(ii) There exist an $\alpha>0$ and a measurable function $h: B_{1} \times \mathbb{R}^{d} \rightarrow[0, \infty)$ with

$$
\sup _{p \in B_{1}}\|h(p, \cdot)\|_{1}<\infty
$$

such that for all $n \in \mathbb{N}$ and almost all $p \in B_{1}$ and $k \in \mathbb{R}^{d}$

$$
\left\|a_{k+p} \psi_{n}-a_{k} \psi_{n}\right\| \leq|p|^{\alpha} h(p, k) .
$$


Proof. (i) follows directly from Lemmas 4.2 and 4.4, and from the monotonicity of $\left(\omega_{n}\right)_{n \in \mathbb{N}}$.

Let $\alpha_{1}$ be the minimum of the values from Hypothesis A (iii) and Hypothesis B (i) and let $\alpha_{2}$ be as in Hypothesis $\mathrm{A}(\mathrm{v})$. Then, we set $\alpha=\min \left\{\alpha_{1}, \alpha_{2}\right\}$ and

$$
\tilde{h}(p, k)=\max \left\{\frac{|f(k+p)-f(k)|}{|p|^{\alpha} \sqrt{\omega(k)}}, \frac{|f(k+p)|}{\omega(k) \sqrt{\omega(k+p)}}\right\} .
$$

Then, by Hypothesis A, $\tilde{h}$ satisfies the above statements on $h$. Further, using the resolvent identity and Lemma 4.2, we obtain

$$
\begin{aligned}
a_{k+p} \psi_{n}-a_{k} \psi_{n}= & f(k) R_{n}(k) \sigma_{x} \psi_{n}-f(k+p) R_{n}(k+p) \sigma_{x} \psi_{n} \\
= & (f(k)-f(k+p)) R_{n}(k) \sigma_{x} \psi_{n} \\
& \quad+f(k+p)\left(R_{n}(k)-R_{n}(k+p)\right) \sigma_{x} \psi_{n} \\
= & (f(k)-f(k+p)) R_{n}(k) \sigma_{x} \psi_{n} \\
& \quad+f(k+p) R_{n}(k)\left(\omega_{n}(k+p)-\omega_{n}(k)\right) R_{n}(k+p) \sigma_{x} \psi_{n} .
\end{aligned}
$$

By Lemma 4.4 and Hypothesis B, we find

$$
|(4.8)| \leq C_{\chi}^{1 / 2} \frac{|f(k+p)-f(k)|}{\sqrt{\omega(k)}} \leq C_{\chi}^{1 / 2}|p|^{\alpha} \tilde{h}(p, k) .
$$

Further, the local $\alpha_{1}$-Hölder continuity of $\omega_{n}$ yields there is $C>0$, such that

$$
|(4.9)| \leq C|p|^{\alpha} \tilde{h}(p, k) .
$$

This proves the statement for the function $h=\left(C_{\chi}^{1 / 2}+C\right) \tilde{h}$.

We use the above infrared bounds to derive an upper bound on the photon number operator and the free field energy

$$
N=\mathbb{1} \otimes \mathrm{d} \Gamma(1) \quad \text { and } \quad H_{\mathrm{f}}=\mathbb{1} \otimes \mathrm{d} \Gamma(\omega)
$$

acting on the ground states $\psi_{n}$. The proof uses the following well-known representation of the quadratic form associated with second quantization operators in terms of pointwise annihilation operators.

Lemma 4.6. Assume $A: \mathbb{R}^{d} \rightarrow[0, \infty)$ is measurable and $\psi \in \mathcal{F}$. Then the map $k \mapsto\left\|A(k)^{1 / 2} a_{k} \psi\right\|$ is in $L^{2}\left(\mathbb{R}^{d}\right)$ if and only if $\psi \in \mathcal{D}\left(\mathrm{d} \Gamma(A)^{1 / 2}\right)$. Further, for any $\phi_{1}, \phi_{2} \in \mathcal{D}\left(\mathrm{d} \Gamma(A)^{1 / 2}\right)$ we have

$$
\left\langle\mathrm{d} \Gamma(A)^{1 / 2} \phi_{1}, \mathrm{~d} \Gamma(A)^{1 / 2} \phi_{2}\right\rangle=\int_{\mathbb{R}^{d}} A(k)\left\langle a_{k} \phi_{1}, a_{k} \phi_{2}\right\rangle \mathrm{d} k .
$$

Proof. The statement is standard in the literature, see for example [31].

The next lemma will provide a photon number bound.

Lemma 4.7. For all $n \in \mathbb{N}$ we have $\psi_{n} \in \mathcal{D}\left(N^{1 / 2}\right) \cap \mathcal{D}\left(H_{\mathrm{f}}\right)$ and the inequalities $\left\langle N^{1 / 2} \psi_{n}, N^{1 / 2} \psi_{n}\right\rangle \leq C_{\chi}\left\|\omega^{-1 / 2} f\right\|^{2}$ and $\left\langle\psi_{n}, H_{\mathrm{f}} \psi_{n}\right\rangle \leq C_{\chi}\|f\|^{2}$.

Proof. The property $\psi_{n} \in \mathcal{D}\left(H_{\mathrm{f}}\right)$ was proven in Lemma 2.2. The remaining statements follow from combining the upper bound in Lemma 4.5(i) and Lemma 4.6. 


\section{The Compactness Argument}

In this section, we construct a compact set $K \subset \mathcal{H}$, such that $\left(\psi_{n}\right)_{n \in \mathbb{N}} \subset K$. We then use the compactness of $K$ to prove Theorem 2.8. Throughout this section, we assume that Hypothesis A and B hold.

Let us begin with the definition of $K$. To that end, assume $y_{i}$ for $i=1, \ldots, \ell$ is the position operator acting on $\psi^{(\ell)} \in \mathcal{F}^{(\ell)}$ as

$$
\widehat{y_{i} \psi^{(\ell)}}\left(x_{1}, \ldots, x_{\ell}\right)=x_{i} \widehat{\psi^{(\ell)}}\left(x_{1}, \ldots, x_{\ell}\right),
$$

where $\widehat{r}$ denotes the Fourier transform. For $\delta>0$, we now define a closed quadratic form $q_{\delta}$ acting on $\phi=\left(\phi_{1}, \phi_{2}\right) \in \mathcal{Q}\left(q_{\delta}\right) \subset \mathcal{H}$ with natural domain as

$$
q_{\delta}(\phi)=\left\langle N^{1 / 2} \phi, N^{1 / 2} \phi\right\rangle+\sum_{\substack{\ell \in \mathbb{N} \\ s \in\{1,2\}}} \frac{1}{\ell^{2}} \sum_{i=1}^{\ell}\left\langle\phi_{s}^{(\ell)},\left|y_{i}\right|^{\delta} \phi_{s}^{(\ell)}\right\rangle+\left\langle H_{\mathrm{f}}^{1 / 2} \phi, H_{\mathrm{f}}^{1 / 2} \phi\right\rangle,
$$

where $N$ and $H_{\mathrm{f}}$ are defined as in (4.10). Now define

$$
K_{\delta, C}:=\left\{\phi \in \mathcal{Q}\left(q_{\delta}\right):\|\phi\| \leq 1, q_{\delta}(\phi) \leq C\right\} \quad \text { for } C>0 .
$$

Lemma 5.1. For all $\delta, C>0$ the set $K_{\delta, C} \subset \mathcal{H}$ is compact.

Proof. By definition, $q_{\delta}$ is nonnegative. Hence, there exists a self-adjoint nonnegative operator $T$ associated to $q_{\delta}$. By the general characterization of operators with compact resolvent (cf. [32, Theorem XIII.64]), $K_{\delta, C}$ is compact iff $T$ has compact resolvent iff the $i$-th eigenvalues of $T$ obtained by the min-max principle $\mu_{i}(T)$ tend to infinity, i.e., $\lim _{i \rightarrow \infty} \mu_{i}(T)=\infty$.

To that end, we observe $T$ preserves the $\ell$ photon sectors $\mathbb{C}^{2} \otimes \mathcal{F}^{(\ell)}$ and denote $T_{\ell}=T\left\lceil\mathbb{C}^{2} \otimes \mathcal{F}^{(\ell)}\right.$. Now, since $(\omega+1)^{(\ell)}(K) \rightarrow \infty$ as $K \rightarrow \infty$ by Hypothesis A (ii), we can apply Rellich's criterion (cf. [32, Theorem XIII.65]) and hence $T_{\ell}$ has compact resolvent for all $\ell \in \mathbb{N}_{0}$. As argued above, we have $\lim _{i \rightarrow \infty} \mu_{i}\left(T_{\ell}\right)=\infty$. Further, since $T_{\ell} \geq \ell$, we have $\mu_{i}\left(T_{\ell}\right) \geq \ell$ and therefore $\lim _{i \rightarrow \infty} \mu_{i}(T)=\infty$.

We now need to prove the following proposition, where $\psi_{n}$ are the normalized ground states of $H_{n}$ as defined in Sect. 3.

Proposition 5.2. There are $\delta, C>0$, such that $\psi_{n} \in K_{\delta, C}$ for all $n \in \mathbb{N}$.

For the proof the following lemma is essential. Hereby, for $n \in \mathbb{N}, s \in\{1,2\}$ and $y, k \in \mathbb{R}^{d}$, we introduce the notation

$$
\begin{aligned}
& \widehat{\psi_{n, s}^{(\ell)}}(y):\left(y_{1}, \ldots, y_{\ell-1}\right) \mapsto \psi_{n, s}^{(\ell)}\left(y, y_{1}, \ldots, y_{\ell-1}\right), \\
& \psi_{n, s}^{(\ell)}(k):\left(k_{1}, \ldots, k_{\ell-1}\right) \mapsto \psi_{n, s}^{(\ell)}\left(k, k_{1}, \ldots, k_{\ell-1}\right) .
\end{aligned}
$$

Due to the Fubini-Tonelli theorem, we have $\widehat{\psi_{n, s}^{(\ell)}}(y), \psi_{n, s}^{(\ell)}(k) \in L^{2}\left(\mathbb{R}^{(\ell-1) d}\right)$ for almost every $k, y \in \mathbb{R}^{d}$. Further, comparing with the definition (4.2), we observe

$$
\psi_{n, s}^{(\ell)}(k)=\frac{1}{\sqrt{\ell+1}}\left(a_{k} \psi_{n, s}\right)^{(\ell)} .
$$


Lemma 5.3. There exist $\delta>0$ and $C>0$, such that for all $p \in \mathbb{R}^{d}$ and $n, \ell \in \mathbb{N}$, $s \in\{1,2\}$

$$
\int_{\mathbb{R}^{d}}\left|1-e^{-i p y}\right|^{2}\left\|\widehat{\psi_{n, s}^{(\ell)}}(y)\right\|_{L^{2}\left(\mathbb{R}^{(\ell-1) d}\right)}^{2} \mathrm{~d} y \leq \frac{C}{\ell+1} \min \left\{1,|p|^{\delta}\right\} .
$$

We note that $\delta$ can be chosen as $\delta=\frac{\epsilon \alpha}{1+\epsilon}$, where $\alpha>0$ and $\epsilon>0$ are as in Lemma 4.5 (ii) and Hypothesis A (iv), respectively.

Proof. That the left hand side of (5.6) is bounded by a constant $C$, uniformly in $p$, follows easily due to the Fock space definition, since the Fourier transform preserves the $L^{2}$-norm. Now lets consider $|p| \leq 1$. Note that

$$
\begin{gathered}
\int_{\mathbb{R}^{d}}\left|1-e^{-i p y}\right|^{2}\left\|\widehat{\psi_{n, s}^{(\ell)}}(y)\right\|_{L^{2}\left(\mathbb{R}^{(\ell-1) d}\right)}^{2} \mathrm{~d} y=\int_{\mathbb{R}^{d}}\left\|\psi_{n, s}^{(\ell)}(k+p)-\psi_{n, s}^{(\ell)}(k)\right\|^{2} \mathrm{~d} k \\
=\frac{1}{\ell+1} \int_{\mathbb{R}^{d}}\left\|\left(a_{k+p} \psi_{n, s}\right)^{(\ell)}-\left(a_{k} \psi_{n, s}\right)^{(\ell)}\right\|^{2} \mathrm{~d} k,
\end{gathered}
$$

where we used (5.5). Let $\theta \in(0,1)$ and write

$$
w(p, k)=\max \left\{\frac{|f(k)|}{\omega(k)^{1 / 2}}, \frac{|f(k+p)|}{\omega(k+p)^{1 / 2}}\right\} .
$$

By Lemma 4.5, we have some $C>0$ such that

$$
\left\|\left(a_{k+p} \psi_{n, s}\right)^{(\ell)}-\left(a_{k} \psi_{n, s}\right)^{(\ell)}\right\| \leq C|p|^{\theta \alpha} h(p, k)^{\theta} w(p, k)^{1-\theta} .
$$

For $r, r^{\prime}>1$ with $\frac{1}{r}+\frac{1}{r^{\prime}}=1$, we now use Young's inequality $b c \leq b^{r} / r+c^{r^{\prime}} / r^{\prime}$ to obtain a constant $C_{r}>0$ with

$$
\left\|\left(a_{k+p} \psi_{n, s}\right)^{(\ell)}-\left(a_{k} \psi_{n, s}\right)^{(\ell)}\right\|^{2} \leq C_{r}|p|^{2 \theta \alpha}\left(h(p, k)^{2 \theta r}+w(p, k)^{2(1-\theta) r^{\prime}}\right) .
$$

Set $r=\frac{1}{2 \theta}$. Then, the first summand in (5.7) is integrable in $k$ due to Lemma 4.5. Further, the exponent of the second summand equals

$$
2(1-\theta) r^{\prime}=2(1-\theta)\left(1-\frac{1}{r}\right)^{-1}=\frac{2(1-\theta)}{1-2 \theta} .
$$

Hence, we can choose $\theta>0$ such that $\frac{2(1-\theta)}{1-2 \theta}=2+\epsilon$. By Hypothesis A (iv), it follows that (5.7) is integrable in $k$ and the proof is complete.

From here, we can prove an upper bound for the Fourier term in (5.2).

Lemma 5.4. Let $\delta>0$ be as in Lemma 5.3. Then there exists $C>0$ such that for all $n, \ell \in \mathbb{N}$ and $s \in\{1,2\}$

$$
\int_{\mathbb{R}^{d \cdot \ell}} \sum_{i=1}^{\ell}\left|x_{i}\right|^{\delta / 2}\left|\widehat{\psi_{n, s}^{(\ell)}}\left(x_{1}, \ldots, x_{\ell}\right)\right|^{2} \mathrm{~d}\left(x_{1}, \ldots, x_{\ell}\right) \leq C .
$$


Proof. From Lemma 5.3, we know that there exists a finite constant $C$ such that

$$
\int_{\mathbb{R}^{d}} \int_{\mathbb{R}^{d}} \frac{\left|1-e^{-i p y}\right|^{2}\left\|\widehat{\psi_{n, s}^{(\ell)}}(y)\right\|^{2}}{|p|^{\delta / 2}} \mathrm{~d} y \frac{\mathrm{d} p}{|p|^{d}} \leq \frac{C}{\ell+1} .
$$

After interchanging the order of integration and a change of integration variables $q=$ $|y| p$, we find

$$
\begin{aligned}
\frac{C}{\ell+1} & \geq \int_{\mathbb{R}^{d}} \widehat{\| \psi_{n, s}^{(\ell)}}(y) \|^{2} \int_{\mathbb{R}^{d}} \frac{\left|1-e^{-i p y}\right|^{2}}{|p|^{\delta / 2}} \frac{\mathrm{d} p}{|p|^{d}} \mathrm{~d} y \\
& =\int_{\mathbb{R}^{d}} \widehat{\| \psi_{n, s}^{(\ell)}}(y) \|^{2}|y|^{\delta / 2} \underbrace{\int_{\mathbb{R}^{d}} \frac{\left|1-e^{-i q y /|y|}\right|^{2}}{|q|^{\delta / 2}} \frac{\mathrm{d} q}{|q|^{d}}}_{=: c} \mathrm{~d} y,
\end{aligned}
$$

where $c$ is nonzero and does not depend on $y$.

We can now conclude.

Proof of Proposition 5.2. Combine Lemmas 4.7 and 5.4.

Proof of Theorem 2.8. By Lemma 5.1 and Proposition 5.2, we know there exists a subsequence $\left(\psi_{n_{k}}\right)_{k \in \mathbb{N}}$, which converges to a normalized vector $\psi_{\infty} \in K_{\delta, C}$. By construction and Lemma 2.2, we have $K_{\delta, C} \subset \mathcal{D}\left(H_{f}^{1 / 2}\right)=\mathcal{D}\left((H-E)^{1 / 2}\right)$. Further, it is known that any closed quadratic form is lower-semicontinuous, i.e., if $x=\lim _{n \rightarrow \infty} x_{n}$ in the domain of a closed form $q$ then $q(x) \leq \liminf _{n \rightarrow \infty} q\left(x_{k}\right)$ (see for example [29, Chapter VI, Theorem 1.16]). Hence, Proposition 3.3 yields

$$
\begin{aligned}
\left\|(H-E)^{1 / 2} \psi_{\infty}\right\|^{2} & =\left\langle(H-E)^{1 / 2} \psi_{\infty},(H-E)^{1 / 2} \psi_{\infty}\right\rangle \\
& \leq \liminf _{k \rightarrow \infty}\left\langle\psi_{n_{k}},(H-E) \psi_{n_{k}}\right\rangle=0 .
\end{aligned}
$$

Hence, $(H-E)^{1 / 2} \psi_{\infty}=0$. This especially implies $(H-E)^{1 / 2} \psi_{\infty} \in \mathcal{D}\left((H-E)^{1 / 2}\right)$, which in turn gives $\psi_{\infty} \in \mathcal{D}(H)$ and yields $H \psi_{\infty}=E \psi_{\infty}$.

Acknowledgements. D.H. wants to thank Ira Herbst for valuable discussions on the subject.

Funding Open Access funding enabled and organized by Projekt DEAL.

Open Access This article is licensed under a Creative Commons Attribution 4.0 International License, which permits use, sharing, adaptation, distribution and reproduction in any medium or format, as long as you give appropriate credit to the original author(s) and the source, provide a link to the Creative Commons licence, and indicate if changes were made. The images or other third party material in this article are included in the article's Creative Commons licence, unless indicated otherwise in a credit line to the material. If material is not included in the article's Creative Commons licence and your intended use is not permitted by statutory regulation or exceeds the permitted use, you will need to obtain permission directly from the copyright holder. To view a copy of this licence, visit http://creativecommons.org/licenses/by/4.0/.

Publisher's Note Springer Nature remains neutral with regard to jurisdictional claims in published maps and institutional affiliations. 


\section{References}

1. Abdesselam, A.: The ground state energy of the massless spin-boson model. Ann. Henri Poincaré 12, 1321-1347 (2011). https://doi.org/10.1007/s00023-011-0103-6

2. Abdesselam, A., Hasler, D.: Analyticity of the ground state energy for massless Nelson models. Commun. Math. Phys. 310(2), 511-536 (2012). https://doi.org/10.1007/s00220-011-1407-6

3. Aizenman, M., Newman, C.M.: Discontinuity of the percolation density in one-dimensional $1 /|x-y|^{2}$ percolation models. Commun. Math. Phys. 107(4), 611-647 (1986). https://doi.org/10.1007/BF01205489

4. Arai, A.: Analysis on Fock Spaces and Mathematical Theory of Quantum Fields. World Scientific, New Jersey (2018). https://doi.org/10.1142/10367

5. Arai, A., Hirokawa, M.: On the existence and uniqueness of ground states of the spin-boson Hamiltonian. Hokkaido Univ. Prepr. Ser. Math. 309, 2-20 (1995). https://doi.org/10.14943/83456

6. Arai, A., Hirokawa, M.: On the existence and uniqueness of ground states of a generalized spin-boson model. J. Funct. Anal. 151, 455-503 (1997). https://doi.org/10.1006/jfan.1997.3140

7. Arai, A., Hirokawa, M., Hiroshima, F.: On the absence of eigenvectors of Hamiltonians in a class of massless quantum field models without infrared cutoff. J. Funct. Anal. 168(2), 470-497 (1999). https:// doi.org/10.1006/jfan.1999.3472

8. Bach, V., Ballesteros, M., Könenberg, M., Menrath, L.: Existence of ground state eigenvalues for the spin-boson model with critical infrared divergence and multiscale analysis. J. Math. Anal. Appl. 453(2), 773-797 (2017). https://doi.org/10.1016/j.jmaa.2017.03.075

9. Bach, V., Chen, T., Fröhlich, J., Sigal, I.M.: The renormalized electron mass in non-relativistic quantum electrodynamics. J. Funct. Anal. 243(2), 426-535 (2007). https://doi.org/10.1016/j.jfa.2006.09.017

10. Bach, V., Fröhlich, J., Sigal, I.M.: Quantum electrodynamics of confined nonrelativistic particles. Adv. Math. 137(2), 299-395 (1998). https://doi.org/10.1006/aima.1998.1734

11. Bach, V., Fröhlich, J., Sigal, I.M.: Renormalization group analysis of spectral problems in quantum field theory. Adv. Math. 137(2), 205-298 (1998). https://doi.org/10.1006/aima.1998.1733

12. Bach, V., Fröhlich, J., Sigal, I.M.: Spectral analysis for systems of atoms and molecules coupled to the quantized radiation field. Commun. Math. Phys. 207(2), 249-290 (1999). https://doi.org/10.1007/ s002200050726

13. Dam, T.N., Hinrichs, B.: Absence of ground states in the renormalized massless translation-invariant Nelson model (2019). ArXiv Preprint, arXiv:1909.07661

14. Dam, T.N., Møller, J.S.: Asymptotics in spin-boson type models. Commun. Math. Phys. 374(3), 13891415 (2020). https://doi.org/10.1007/s00220-020-03685-5

15. Dyson, F.J.: Existence of a phase-transition in a one-dimensional Ising ferromagnet. Commun. Math. Phys. 12(2), 91-107 (1969). https://doi.org/10.1007/BF01645907

16. Folland, G.B.: Real Analysis: Modern Techniques and Their Applications, 2nd edn. Pure and Applied Mathematics. Wiley, New York (1999)

17. Fröhlich, J.: On the infrared problem in a model of scalar electrons and massless scalar bosons. Ann. de l'Inst. Henri Poincaré 19(1), 1-103 (1973)

18. Gérard, C.: On the existence of ground states for massless Pauli-Fierz Hamiltonians. Ann. Henri Poincaré 1(3), 443-459 (2000). https://doi.org/10.1007/s000230050002

19. Griesemer, M., Hasler, D.G.: Analytic perturbation theory and renormalization analysis of matter coupled to quantized radiation. Ann. Henri Poincaré 10(3), 577-621 (2009). https://doi.org/10.1007/s00023-0090417-9

20. Griesemer, M., Lieb, E., Loss, M.: Ground states in non-relativistic quantum electrodynamics. Invent. Math. 145(3), 557-595 (2001). https://doi.org/10.1007/s002220100159

21. Hasler, D., Herbst, I.: Absence of ground states for a class of translation invariant models of non-relativistic QED. Commun. Math. Phys. 279(3), 769-787 (2008). https://doi.org/10.1007/s00220-008-0444-2

22. Hasler, D., Herbst, I.: Convergent expansions in non-relativistic QED: analyticity of the ground state. J. Funct. Anal. 261(11), 3119-3154 (2011). https://doi.org/10.1016/j.jfa.2011.07.023

23. Hasler, D., Herbst, I.: Ground states in the spin boson model. Ann. Henri Poincaré 12(4), 621-677 (2011). https://doi.org/10.1007/s00023-011-0091-6

24. Hasler, D., Herbst, I.: Smoothness and analyticity of perturbation expansions in QED. Adv. Math. 228(6), 3249-3299 (2011). https://doi.org/10.1016/j.aim.2011.08.007

25. Hasler, D., Hinrichs, B., Siebert, O.: Correlation bound for a one-dimensional continuous long-range Ising model (2021). ArXiv Preprint arXiv:2104.03013

26. Hasler, D., Hinrichs, B., Siebert, O.: FKN formula and ground state energy for the spin boson model with external magnetic field (2021). ArXiv Preprint arXiv:2106.08659

27. Hasler, D., Siebert, O.: Ground states for translationally invariant Pauli-Fierz models at zero momentum (2020). ArXiv Preprint arXiv:2007.01250 
28. Imbrie, J.Z., Newman, C.M.: An intermediate phase with slow decay of correlations in one-dimensional $1 /|x-y|^{2}$ percolation, Ising and Potts models. Commun. Math. Phys. 118(2), 303-336 (1988). https:// doi.org/10.1007/BF01218582

29. Kato, T.: Perturbation Theory for Linear Operators, Classics in Mathematics, 2nd edn, vol. 132. Springer, Berlin (1980). https://doi.org/10.1007/978-3-642-66282-9

30. Lőrinczi, J., Minlos, R.A., Spohn, H.: The infrared behaviour in Nelson's model of a quantum particle coupled to a massless scalar field. Ann. Henri Poincaré 3, 269-295 (2002). https://doi.org/10.1007/ s00023-002-8617-6

31. Reed, M., Simon, B.: Fourier Analysis, Self-Adjointness, Methods of Modern Mathematical Physics, vol. 2. Academic Press, San Diego (1975)

32. Reed, M., Simon, B.: Analysis of Operators, Methods of Modern Mathematical Physics, vol. 4. Academic Press, San Diego (1978)

33. Spohn, H.: Ground state(s) of the spin-boson Hamiltonian. Commun. Math. Phys. 123(2), 277-304 (1989). https://doi.org/10.1007/BF01238859

34. Spohn, H.: Ground state of a quantum particle coupled to a scalar Bose field. Lett. Math. Phys. 44(1), 9-16 (1998). https://doi.org/10.1023/A:1007473300274

35. Spohn, H., Dümcke, R.: Quantum tunneling with dissipation and the Ising model over $r$. J. Stat. Phys. 41(3), 389-423 (1985). https://doi.org/10.1007/BF01009015

Communicated by H. Spohn 